\title{
San Luis Potosi
}

National Cancer Institute

\section{Source}

National Cancer Institute. San Luis Potosi. NCI Thesaurus. Code C106157.

A state in central Mexico. Its capital is San Luis Potosi. 